\title{
Pediatric In-Hospital Cardiac Arrest
} International Registry (PACHIN): protocol for a prospective international multicenter register of cardiac arrest in children

Jimena del Castillo ${ }^{1,2,3^{*}} \mathbb{D}$, Débora Sanz ${ }^{1,2,3}$, Laura Herrera ${ }^{1,2,3}$ and Jesús López-Herce ${ }^{1,2,3}$ Grupo de Estudio de Parada Cardiaca Intrahospitalaria en la Infancia

\begin{abstract}
Background and aims: Cardiac arrest (CA) in children is a major public health problem. Thanks to advances in cardiopulmonary resuscitation (CPR) guidelines and teaching skills, results in children have improved. However, pediatric CA has a very high mortality. In the treatment of in-hospital CA there are still multiple controversies. The objective of this study is to develop a multicenter and international registry of in-hospital pediatric cardiac arrest including the diversity of management in different clinical and social contexts. Participation in this register will enable the evaluation of the diagnosis of CA, CPR and post-resuscitation care and its influence in survival and neurological prognosis.

Methods: An intrahospital CA data recording protocol has been designed following the Utstein model. Database is hosted according to European legislation regarding patient data protection. It is drafted in English and Spanish. Invitation to participate has been sent to Spanish, European and Latinamerican hospitals. Variables included, asses hospital characteristics, the resuscitation team, patient's demographics and background, CPR, post-resuscitation care, mortality, survival and long-term evolution. Survival at hospital discharge will be evaluated as a primary outcome and survival with good neurological status as a secondary outcome, analyzing the different factors involved in them. The study design is prospective, observational registry of a cohort of pediatric CA.
\end{abstract}

Conclusions: This study represents the development of a registry of in-hospital CA in childhood. Its development will provide access to CPR data in different hospital settings and will allow the analysis of current controversies in the treatment of pediatric CA and post-resuscitation care. The results may contribute to the development of further international recommendations.

Trial register: ClinicalTrials.gov Identifier: NCT04675918. Registered 19 December 2020 - Retrospectively registered, https://clinicaltrials.gov/ct2/show/record/NCT04675918?cond=pediatric+cardiac+arrest\&draw=2\&rank=10

Keywords: Pediatric cardiac arrest, Cardiac arrest, Resuscitation, In-hospital, Clinical registry

*Correspondence: jimenadelcastillo@yahoo.es

1 Pediatric Intensive Care Unit, Gregorio Marañón General University

Hospital, Condado de Treviño 9, 28033 Madrid, Spain

Full list of author information is available at the end of the article

\section{Background}

Pediatric in-hospital cardiac arrest (IHCA) is a rare event with possible devastating consequences. It is considered a major public healthcare problem. Thanks to advances in cardiopulmonary resuscitation (CPR) guidelines and teaching skills, results in children have improved $[1,2]$. 
However, pediatric CA still has a very high mortality [3-5].

Survival to pediatric IHCA has been related to multiple factors. Previous studies have described the influence of patient characteristics, quality of CPR, post-resuscitation factors and team interventions in mortality [3, 4, 6-8]. Current recommendations on CPR have insisted on the importance of quality and post-resuscitation care in an attempt to improve outcomes [9]. However, in the treatment of in-hospital CA there are still multiple controversies and gaps of knowledge.

\section{Quality CPR}

Some clinical and experimental studies have suggested that quality of cardiopulmonary resuscitation (frequency and depth of chest compressions, compression-decompression ratio, coordination of chest compressions and ventilation with coordination of rescuers) is associated with better CPR results [10].

\section{Ventilation for pediatric $C A$}

In the last decade, some authors have suggested that initial basic CPR with only chest compressions may be equal to or better than providing chest compressions plus ventilation. In contrast, clinical studies in children [11] and experimental studies in child animal models [12] have shown that early ventilation and oxygenation are essential in child CPR. Intubation during CPR has for many years been considered an essential maneuver during advanced CPR, as it achieves safe isolation of the airway and allows good ventilation. However, intubation is a technique that requires learning and training and can be difficult to perform in emergency situations such as a CA. In recent years, several studies in adults and children have suggested that intubation during CPR is associated with a worse survival and neurological prognosis [13, 14], but a recent large study in adults does not confirm these results [15]. There are no controlled, experimental, descriptive or clinical studies that have looked at this problem.

\section{Post-resuscitation factors}

Once ROSC is achieved, efforts towards survival have to assess and treat post-cardiac arrest syndrome. Outcomes in terms not only of survival, but of good neurological outcome, depend on various factors, including hemodynamics, ventilation and oxygenation, temperature control, sedation and analgesia.

Hypotension after ROSC is associated with a worse prognosis. The ILCOR group recommends keeping systolic blood pressure (BP) above the 5th percentile, but there are no studies that assess which is the best BP in the post-resuscitation period or whether treating low blood pressure influences outcomes [16].

Several studies in adults and children have analyzed the influence of ventilation and oxygenation after ROSC on prognosis. Although the results between all the studies are not in agreement, most suggest that both hypoventilation and hyperventilation are associated with a worse prognosis [17]. Hypoxia can also be a poor prognostic indicator, while the results with hyperoxia are contradictory [16].

Hyperthermia after ROSC is common and is associated with a worse neurological prognosis, which increases with each degree of body temperature above $37{ }^{\circ} \mathrm{C}$. Experimental studies in animals have demonstrated the neuroprotective effect of hypothermia. Initial studies in adults found that hypothermia improved the neurological prognosis in patients recovered from CA. However, the most recent studies in adults and children have found that therapeutic hypothermia is not associated with a better neurological prognosis or longer survival [18]. Therefore, at the present time, strict temperature control and avoiding hyperthermia are recommended.

Sedation and analgesia are fundamental in caring for the critical care child. They enable anxiety and pain treatment as well as shivering control for temperature management. However, after CA its use can difficult neurological assessment, and to date, no study has been able to describe the influence of different analgosedation protocols in neurological outcomes.

\section{Rational for the study}

For the past years, the International Liaison Committee on Resuscitation (ILCOR) has endeavored its efforts in the identification of these gaps of knowledge and their resolution. There are few clinical data to verify the efficacy of CPR interventions in the pediatric population. Thus, the development of collaborative multicenter studies is needed. The objective of this study is to develop a multicenter and international registry of in-hospital pediatric cardiac arrest including the diversity of management in different clinical and social contexts. Participation in this register will enable the evaluation of the diagnosis of CA, CPR and post-resuscitation care and its influence in survival and neurological prognosis.

\section{Study aims}

The objective of PACHIN is (1) to study the relationship between the characteristics of cardiopulmonary resuscitation (CPR) and outcomes, focusing on the influence of ventilation parameters (intubation or not during resuscitation and controlled or uncontrolled ventilation) on the recovery of spontaneous circulation, survival, and short- and long-term neurological prognosis of children 
with in-hospital cardiac arrest. (2) To study the relationship between post-resuscitation measures (especially ventilation, oxygenation, blood pressure, analgosedation and temperature) with survival and short- and long-term neurological prognosis in children with IHCA. (3) To create a permanent register of pediatric in-hospital cardiac arrest for European and Latinamerican countries.

\section{Methods and analysis Study design}

This study is a multicenter, international, prospective observational registry.

\section{Setting}

Patients will be enrolled by participating investigators from European and Latinamerican countries. All sites are susceptible of treating pediatric cardiac arrest patients. Participating hospitals differ in levels of care but are all able to submit their data to the study's database.

\section{Patient elegibility}

Inclusion criteria: all children aged 1 month to 18 years who suffer a CA in hospital. For the study, CA is defined as the absence of vital signs requiring at least one minute of chest compressions. Subsequent episodes of CA may be included for the same individual.

Exclusion criteria: Patients being treated with extracorporeal circulatory support (ECMO or ventricular assistance) at the time of CA. Patients who suffer a cardiac arrest and require ECMO for ROSC, after performing conventional CPR, will not be excluded. Duration of the data collection period 24 months.

\section{Recruitment}

Study candidates will be identified by a study physician, who will explain the study to parents or guardians. Written informed consent will be obtained from parents or guardians prior to inclusion in the study. A CONSORT (Consolidated Standard of Reporting Trials) flow diagram is shown in Fig. 1.

\section{Data collection}

Data will be collected, verified, and uploaded to a protected electronic web-based database (Xolomon) by their designated site coordinators/investigators under the oversight of their IRB. Database has been designed following the Utstein model [19]. Database is hosted according to European legislation regarding patient data protection. It is drafted in English and Spanish.

Variables recorded will include hospital characteristics, the resuscitation team, patient's demographics and background, CPR, post-resuscitation care, mortality, survival and long-term evolution (see Table 1).

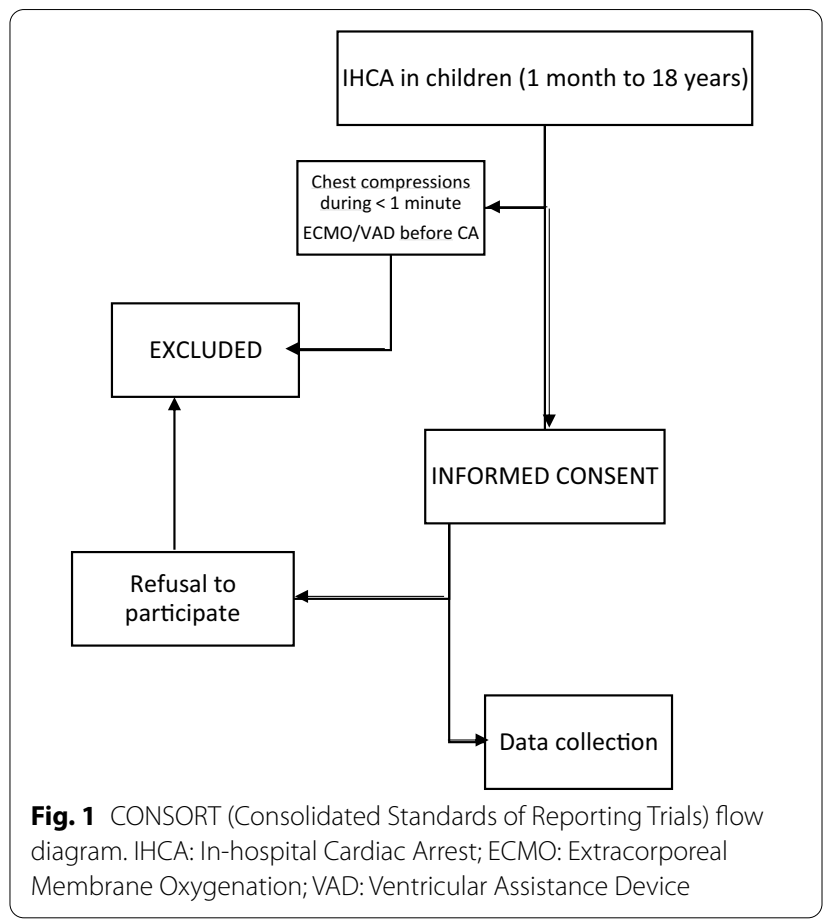

\section{Outcomes assessment}

The primary outcome is survival at hospital discharge. Secondary outcome being survival with favorable neurological status. Analysis of different factors influencing both outcomes will be performed.

\section{Statistical analysis}

Data analysis plan: The data will be manually reviewed for errors, missing data and outliers before analysis. Extreme values will be set to missing if they are deemed unlikely, based on their validity range. Descriptive analysis of the data will be reported. Continuous variables will be reported as either median and interquartile range (IQR) or mean and standard deviation (SD) based on the distribution. Categorical variables will be described in numbers, percentages or both.

\section{Sample size}

As this is an observational study with no intervention, we have not conducted a power calculation. Based on previous studies [3], we estimate that we will enroll at least 250 to 300 eligible cardiac arrest events. This would represent a large international dataset of pediatric cardiac arrest data with significant power to enable exploration of important correlations between performance and survival through multivariate analysis. 
Table 1 Description of variables collected in database

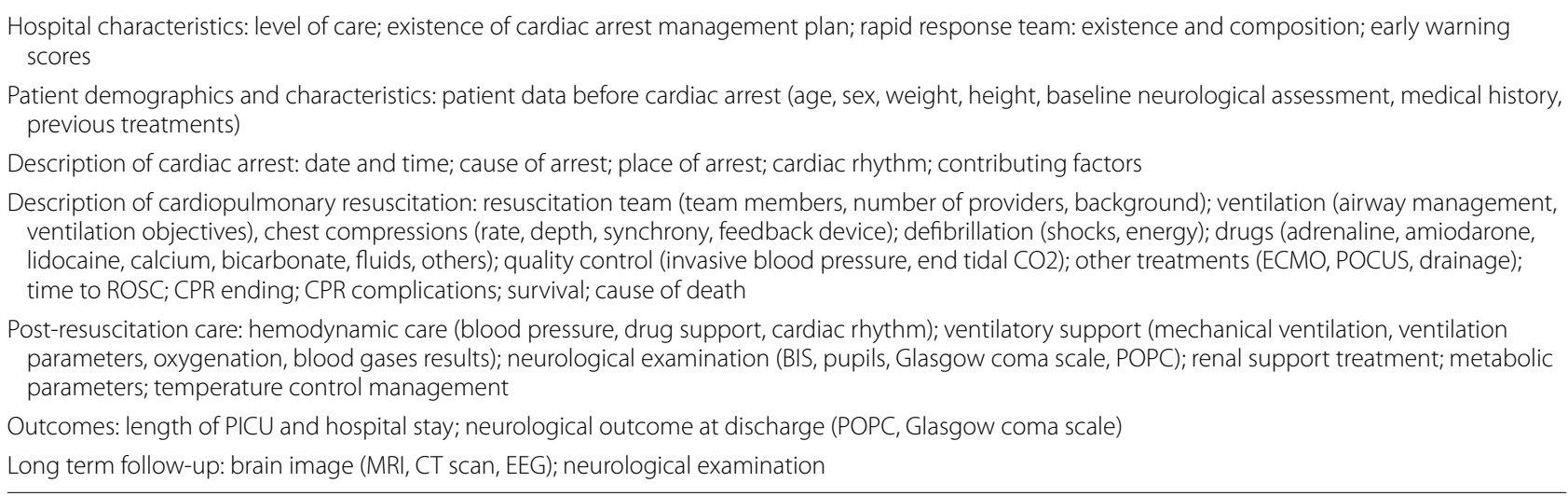

\section{Discussion}

Multicenter collaborative studies are needed in order to assess the gaps in knowledge surrounding pediatric cardiac arrest. Our group as well as others, have developed collaborative platforms to this effect, demonstrating that this approach is feasible and useful [3, 20-22]. These experiences have identified those areas that need further assessment. This is the reason for the creation of a registry of in-hospital CA in childhood in European and Latinamerican countries. Its development will provide access to CPR data in different hospital settings. This data gathering from different countries and hospitals might enlighten the analysis of compliance with the actual guidelines in these countries and the identification of barriers in treatment of pediatric cardiac arrest. Multicenter international collaboration in Spanish and English will allow the investigators to recruit more patients and enable the analysis of current controversies in the treatment of pediatric CA and post-resuscitation care. We expect the results to contribute to the development of further international recommendations.

\section{Limitations}

The main limitation of the study is that patient recruitment cannot be planned because CA is an unexpected sudden event. On the other hand, when CA happens, all attention is driven towards CPR, so it might be difficult to record all data related to the characteristics of CPR while performing it. Another limitation of the study is that the final results of CPR are influenced by many variables and it can be very difficult to differentiate the isolated effect of each factor. Furthermore, recording as many variables as aimed per $\mathrm{CA}$ requires a lot of dedication and careful review and supervision of each case by the study coordinator. Being CA a rare event in pediatric patients, it might be difficult to find statistically significant differences in some parameters such as mortality. Thus, the participation of a significant number of hospital centers and collaborating researchers is necessary. This is one of the fundamental reasons for a multicenter study. Finally, the neurological follow-up at one year of some patients will end after the end of the study period.

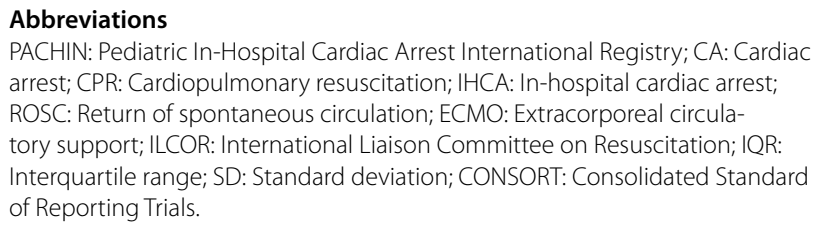
arrest; CPR: Cardiopulmonary resuscitation; IHCA: In-hospital cardiac arrest; ROSC: Return of spontaneous circulation; ECMO: Extracorporeal circulatory support; ILCOR: International Liaison Committee on Resuscitation; IQR: Interquartile range; SD: Standard deviation; CONSORT: Consolidated Standard of Reporting Trials.

\section{Acknowledgements \\ Grupo Español de Estudio de Parada Cardíaca en la Infancia: Cristina Calvo, Carmen Campos, Sonia Cañadas, Juan Carlos de Carlos, Maria Concepción Goñi, Ramón Hernández, Ana Jordá, Juan Mayordomo, Abián Montesdeoca, Antonio Morales, Sara Moralo, Ana Muñoz, Aránzazu Olloqui, Antonio Rodri- guez, María Luisa Serrano.}

\section{Authors' contributions}

$\mathrm{JdC}$ and JLH have been responsible for the conception and design of the protocol. DB ans LH have contributed to the creation of the database and acquisition of data. All authors read and approved the final manuscript.

\section{Funding}

The author(s) disclosed receipt of the following financial support for the research of this article: This work was supported by the Instituto de Salud Carlos III through the project "PI18/01632" (Co-funded by European Regional Development Fund/European Social Fund "A way to make Europe"/"Investing in your future"). The funders, through the grant granted, support the costs of the design of the database, data collection and analysis.

\section{Availability of data and materials}

Not applicable.

\section{Declarations}

\section{Ethics approval and consent to participate}

Approval for study development was obtained from the Coordinating center Ethics Committee (Gregorio Marañón Hospital Ethics Committee CPMP/ $\mathrm{ICH} / 135 / 95)$. Informed consent from patient's guardians is obtained through a written form. 


\section{Consent for publication \\ Not applicable.}

\section{Competing interests}

The authors declare that they have no competing interests.

\section{Trial status}

Protocol version number and date: PACHIN 1, September 2020. Recruitment began he date recruitment began October 2020. Recruitment will be completed October 2025.

\section{Author details}

1 Pediatric Intensive Care Unit, Gregorio Marañón General University Hospital, Condado de Treviño 9, 28033 Madrid, Spain. ${ }^{2}$ Gregorio Marañón Health Research Institute (IISGM), Madrid, Spain. ${ }^{3}$ Maternal and Child Health and Development Research Network (REDSAMID), Institute of Health Carlos III, Madrid, Spain.

Received: 15 June 2021 Accepted: 21 July 2021

Published online: 31 July 2021

\section{References}

1. Girotra S, Spertus JA, Li Y, Berg RA, Nadkarni VM, Chan PS, et al. Survival trends in pediatric in-hospital cardiac arrests: an analysis from Get With the Guidelines-Resuscitation. Circ Cardiovasc Qual Outcomes. 2013:6(1):42-9.

2. Holmberg MJ, Wiberg S, Ross CE, Kleinman M, Hoeyer-Nielsen AK, Donnino MW, et al. Trends in survival after pediatric in-hospital cardiac arrest in the United States. Circulation. 2019;140(17):1398-408.

3. López-Herce J, Del Castillo J, Matamoros M, Cañadas S, Rodriguez-Calvo $A$, Cecchetti C, et al. Factors associated with mortality in pediatric in-hospital cardiac arrest: a prospective multicenter multinational observational study. Intensive Care Med. 2013;39(2):309-18.

4. López-Herce J, del Castillo J, Matamoros M, Canadas S, Rodriguez-Calvo A, Cecchetti C, et al. Post return of spontaneous circulation factors associated with mortality in pediatric in-hospital cardiac arrest: a prospective multicenter multinational observational study. Crit Care Lond Engl. 2014;18(6):607.

5. Lopez-Herce J, Del Castillo J, Carrillo A. Cardiac arrest prognostic factors in children. Resuscitation. 2014;85(3):e35.

6. Lin Y-R, Li C-J, WU T-K, Chang Y-J, Lai S-C, Liu T-A, et al. Post-resuscitative clinical features in the first hour after achieving sustained ROSC predict the duration of survival in children with non-traumatic out-of-hospital cardiac arrest. Resuscitation. 2010;81(4):410-7.

7. Topjian AA, French B, Sutton RM, Conlon T, Nadkarni VM, Moler FW, et al Early postresuscitation hypotension is associated with increased mortality following pediatric cardiac arrest. Crit Care Med. 2014;42(6):1518-23.

8. Sutton RM, Reeder RW, Landis WP, Meert KL, Yates AR, Morgan RW, et al. Ventilation rates and pediatric in-hospital cardiac arrest survival outcomes. Crit Care Med. 2019;47(11):1627-36.

9. Maconochie IK, Bingham R, Eich C, López-Herce J, Rodríguez-Núñez A, Rajka T, et al. European resuscitation council guidelines for resuscitation 2015: section 6. Paediatric life support. Resuscitation. 2015;95:223-48.
10. Sutton RM, Wolfe H, Nishisaki A, Leffelman J, Niles D, Meaney PA, et al. Pushing harder, pushing faster, minimizing interruptions... but falling short of 2010 cardiopulmonary resuscitation targets during in-hospital pediatric and adolescent resuscitation. Resuscitation. 2013;84(12):1680-4.

11. Kitamura T, Iwami T, Kawamura T, Nagao K, Tanaka H, Nadkarni VM, et al. Conventional and chest-compression-only cardiopulmonary resuscitation by bystanders for children who have out-of-hospital cardiac arrests: a prospective, nationwide, population-based cohort study. Lancet Lond Engl. 2010;375(9723):1347-54.

12. Botran M, Lopez-Herce J, Urbano J, Solana MJ, Garcia A, Carrillo A. Chest compressions versus ventilation plus chest compressions: a randomized trial in a pediatric asphyxial cardiac arrest animal model. Intensive Care Med. 2011:37(11):1873-80.

13. Andersen LW, Raymond TT, Berg RA, Nadkarni VM, Grossestreuer AV, Kurth T, et al. Association between tracheal intubation during pediatric in-hospital cardiac arrest and survival. JAMA. 2016;316(17):1786-97.

14. Andersen LW, Granfeldt A, Callaway CW, Bradley SM, Soar J, Nolan JP, et al. Association between tracheal intubation during adult in-hospital cardiac arrest and survival. JAMA. 2017;317(5):494-506.

15. Jabre P, Penaloza A, Pinero D, Duchateau F-X, Borron SW, Javaudin F, et al. Effect of bag-mask ventilation vs endotracheal intubation during cardiopulmonary resuscitation on neurological outcome after outof-hospital cardiorespiratory arrest: a randomized clinical trial. JAMA. 2018;319(8):779-87.

16. Topjian AA, de Caen A, Wainwright MS, Abella BS, Abend NS, Atkins DL, et al. Pediatric post-cardiac arrest care: a scientific statement from the american heart association. Circulation. 2019;140(6):e194-233.

17. Del Castillo J, López-Herce J, Matamoros M, Cañadas S, Rodriguez-Calvo A, Cechetti C, et al. Hyperoxia, hypocapnia and hypercapnia as outcome factors after cardiac arrest in children. Resuscitation. 2012;83(12):1456-61.

18. Moler FW, Silverstein FS, Holubkov R, Slomine BS, Christensen JR, Nadkarni VM, et al. Therapeutic hypothermia after in-hospital cardiac arrest in children. N Engl J Med. 2017;376(4):318-29.

19. Suominen P, Olkkola KT, Voipio V, Korpela R, Palo R, Räsänen J. Utstein style reporting of in-hospital paediatric cardiopulmonary resuscitation. Resuscitation. 2000;45(1):17-25.

20. Del Castillo J, López-Herce J, Matamoros M, Cañadas S, Rodríguez-Calvo A, Cecchetti C, et al. Long-term evolution after in-hospital cardiac arrest in children: prospective multicenter multinational study. Resuscitation. 2015:96:126-34.

21. Niles DE, Duval-Arnould J, Skellett S, Knight L, Su F, Raymond TT, et al. Characterization of pediatric in-hospital cardiopulmonary resuscitation quality metrics across an international resuscitation collaborative. Pediatr Crit Care Med J Soc Crit Care Med World Fed Pediatr Intensive Crit Care Soc. 2018;19(5):421-32.

22. Sweberg T, Sen Al, Mullan PC, Cheng A, Knight L, Del Castillo J, et al. Description of hot debriefings after in-hospital cardiac arrests in an international pediatric quality improvement collaborative. Resuscitation. 2018;128:181-7.

\section{Publisher's Note}

Springer Nature remains neutral with regard to jurisdictional claims in published maps and institutional affiliations.

Ready to submit your research? Choose BMC and benefit from:

- fast, convenient online submission

- thorough peer review by experienced researchers in your field

- rapid publication on acceptance

- support for research data, including large and complex data types

- gold Open Access which fosters wider collaboration and increased citations

- maximum visibility for your research: over $100 \mathrm{M}$ website views per year

At BMC, research is always in progress.

Learn more biomedcentral.com/submissions 